\title{
NLRP3 inflammasome expression in peripheral blood monocytes of coronary heart disease patients and its modulation by rosuvastatin
}

\author{
JIAN ZHU, SHILI WU, SIGAN HU, HUI LI, MIAONAN LI，XU GENG and HONGJU WANG
}

Department of Cardiology, The First Affiliated Hospital of Bengbu Medical College, Bengbu, Anhui 233004, P.R. China

Received November 6, 2018; Accepted May 29, 2019

DOI: $10.3892 / \mathrm{mmr} .2019 .10382$

\begin{abstract}
Nucleotide-binding oligomerization domain, leucine rich repeat, and pyrin domain-containing protein 3 (NLRP3) inflammasome has been implicated in a series of physiological and pathological processes. However, its correlation in coronary heart disease (CHD) still remains to be elucidated. The present study aimed to determine the expression of NLRP3 inflammasome in peripheral blood monocytes (PBMCs) of stable angina pectoris (SAP) and acute myocardial infarction (AMI) patients. In addition, the effect of rosuvastatin on their activities was analyzed in vitro. A total of 60 participants with SAP $(n=20)$, AMI $(n=20)$ and non-CHD controls $(n=20)$ were enrolled. Fluorescence-activated cell sorting, real-time PCR, western blotting and enzyme-linked immunosorbent assay were performed to reveal the role of NLRP3 inflammasome. NLRP3 inflammasome was expressed in the PBMCs, and revealed an increased expression along the downstream interleukin (IL)-1 $\beta$ and IL-18 in both SAP and AMI groups, compared to the control group. Moreover, there was a more marked increase in the expression of these indicators in AMI patients when compared to SAP patients. Interference with rosuvastatin in vitro revealed that the expression of NLRP3 inflammasome and its downstream cytokines were significantly downregulated in both SAP and AMI groups in a time-dependent manner. The activation of NLRP3 inflammasome may be involved in the development of CHD, and rosuvastatin could attenuate the inflammatory process of atherosclerosis by downregulating NLRP3 expression and its downstream mediators. These findings indicated a potential role of NLRP3 in the pathogenesis and management of CHD, and also provided new insights into the mechanistic framework of rosuvastatin activity.
\end{abstract}

Correspondence to: Dr Hongju Wang, Department of Cardiology, The First Affiliated Hospital of Bengbu Medical College, 287 Changhuai Road, Bengbu, Anhui 233004, P.R. China

E-mail: hongjuwang@outlook.com

Key words: NLRP3 inflammasome, stable angina pectoris, acute myocardial infarction, rosuvastatin, inflammatory pathogenesis

\section{Introduction}

Coronary heart disease (CHD) with atherosclerosis predominantly affects the inflammatory process, and remains to be a major cause of morbidity and mortality worldwide (1). Several clinical studies are currently underway in assessing various anti-inflammatory agents for the treatment of atherosclerosis (2). Recently, the CANTOS trial has demonstrated that anti-inflammatory therapy targeting the interleukin (IL)- $1 \beta$ innate immunity pathway reduced the risk of recurrent cardiovascular events in patients with prior heart attack (3). In addition, their previous findings indicated that high-sensitivity C-reactive protein (hs-CRP) and IL-6 are associated with an increased risk of cardiovascular events, independent of the cholesterol level (4). Although the specific mechanisms are unclear, several studies have underlined the central role of inflammasomes, which act as integral parts of the innate immune system during the process of atherosclerosis (5-7).

Nucleotide-binding oligomerization domain, leucine rich repeat, and pyrin domain-containing protein 3 (NLRP3), apoptosis-associated speck-like protein containing a CARD (ASC) and caspase-1 constitute the main components of inflammasome-NLRP3 inflammasome (8). The NLRP3 inflammasome on activation forms a macromolecular protein complex that regulates the activation of caspase-1 and production and maturation of pro-inflammatory cytokines such as IL-1 $\beta$ and IL-18 (9). It can be activated by various signals, wherein the cholesterol crystals act as a major trigger and as a sustaining factor for atherogenic inflammation through NLRP3 inflammasome and its induction of IL-1 $\beta$ (10).

Clinically, statins [HMG-CoA (3-hydroxy-3-methylglutaryl-coenzyme A) reductase inhibitors] have long been used for the treatment of CHD $(11,12)$. Statins can effectively stabilize or reverse atherosclerotic plaque formation, improving prognosis, and reducing mortality and morbidity by lowering the blood lipid levels and inhibiting the inflammatory response (13). Rosuvastatin is a relatively new drug that belongs to HMG-CoA inhibitors. It has a safety and tolerability profile similar to or better than that of commonly used doses of other statins (14). Rosuvastatin is associated with a decrease in the levels of serum hs-CRP, which occurs independently with the reduction of low-density lipoprotein cholesterol (LDL-C) (15). Therefore, it is generally used in clinics to prevent and treat CHD (16). However, research regarding the 
correlation between NLRP3 inflammasome and rosuvastatin is hardly available.

Thus, the present study aimed to determine whether NLRP3 inflammasome was expressed in peripheral blood monocytes (PBMCs) and correlated with IL-1 $\beta$ and IL-18 secretion in stable angina pectoris (SAP) and acute myocardial infarction (AMI) patients. In addition, the effect of rosuvastatin on the NLRP3 inflammasome signaling pathway was investigated in $\mathrm{CHD}$ in vitro by utilizing a cell culture model to provide new insights into the inflammatory pathogenesis and management of CHD.

\section{Materials and methods}

Study population. A total of 40 patients with stable SAP $(n=20)$ and AMI $(n=20)$ admitted to our hospital for coronary angiography and emergency percutaneous coronary intervention (PCI) from May 2017 to April 2018 were enrolled. None of the participants were treated with statins before. SAP was defined according to the ACCF/AHA/ACP/AATS/PCNA/SCAI/STS Guidelines (17). AMI was diagnosed based on the ESC/ACCF/AHA/WHF Third Universal Definition of myocardial infarction (18). Patients with clinical signs of acute infection, severe renal failure (serum creatine levels $>3 \mathrm{mg} / \mathrm{dl}$ ) or rheumatoid disease, or suspected of having a malignant or primary wasting disorder were excluded from the present study. A total of 20 subjects with paroxysmal supraventricular tachycardia (PSVT) admitted to our hospital for radiofrequency ablation (RFCA) and exhibiting no evidence of CHD upon history or physical examination were defined as the control group (non-CHD group). Participants included in our study were aged between 41 and 84 years, and utilization of samples was approved by the Ethics Committee of The First Affiliated Hospital of Bengbu Medical College (Bengbu, China) and written informed consent was obtained from all subjects.

Blood samples. Peripheral blood samples were collected from all patients in the morning after an overnight fast to obtain baseline data. Plasma was separated by centrifugation at $2,500 \mathrm{x} \mathrm{g}$ for $15 \mathrm{~min}$ and was stored at $-80^{\circ} \mathrm{C}$ until use.

Cell preparation. Peripheral blood mononuclear cells were isolated from peripheral blood samples by Ficoll-Paque density gradient centrifugation using lymphocyte separation medium (Sigma-Aldrich; Merck KGaA). Isolated mononuclear cells were washed and centrifuged twice with PBS lotion containing 3\% fetal bovine serum (FBS) and $10 \mathrm{mM}$ EDTA for $15 \mathrm{~min}$ at $400 \mathrm{x} \mathrm{g}$ at room temperature. Subsequently, the peripheral blood mononuclear cells were resuspended at a final concentration of $1.0 \times 10^{8}$ cells $/ \mathrm{ml}$ and were isolated using EasySep $^{\mathrm{TM}} \mathrm{CD} 4^{+}$antibody positive selection kit (Stemcell Technologies, Inc.). The obtained PBMCs were washed with PBS lotion containing 3\% FBS and $10 \mathrm{mM}$ EDTA three times. A final concentration of $5.0 \times 10^{6}$ cells $/ \mathrm{ml}$ of PBMCs were incubated with $2.5 \mu \mathrm{l}$ PE anti-human CD14 antibody (1:1,000; cat. no. 12-0149-41; eBioscience; Thermo Fisher Scientific, Ltd.) at $4^{\circ} \mathrm{C}$ for 30 min protected from light. A monoclonal antibody against mouse IgG (1:1,000; cat. no. 10400C; Invitrogen; Thermo Fisher Scientific, Ltd.) was used as the homologous control under the same conditions. After washing thrice with PBS, the cells were resuspended and the positive rate of CD14 was calculated by flow cytometry.

Cell experiments. PBMCs from each group were cultured and proliferated with RPMI-1640 culture medium containing $10 \%$ FBS (both from Thermo Fisher Scientific, Inc.) and $50 \mathrm{U} / \mathrm{ml}$ rIL-2 (Sigma-Aldrich; Merck KGaA) for $24 \mathrm{~h}$ in vitro. Then, the PBMCs were harvested and equally divided into three portions. One portion was defined as the untreated group $(0 \mathrm{~h})$ and collected directly for RNA and protein extraction. The other two portions were sequentially cultured with rosuvastatin for another 12 and $24 \mathrm{~h}$, respectively, and were defined as the 12 and 24-h groups. Rosuvastatin (Sigma-Aldrich; Merck $\mathrm{KGaA}$ ) was dissolved in DMSO at a stock solution of $20 \mathrm{mM}$ and stored in aliquots at $-20^{\circ} \mathrm{C}$ as previously described (19).

RNA isolation and real-time reverse transcriptase-polymerase chain reaction ( $R T-P C R)$. Total RNA was extracted using TRIzol reagent (Invitrogen; Thermo Fisher Scientific, Inc.) according to the manufacturer's instructions. M-MLV reverse transcriptase (Promega Corporation) was used to convert RNA into cDNA at $42^{\circ} \mathrm{C}$ for 60 min following standard procedure. For real-time reverse transcriptase-polymerase chain reaction (RT-PCR) quantification, $2 \mu \mathrm{l}$ of cDNA reaction was amplified in a $20-\mu 1$ standard RT-PCR reaction. The PCR parameters were as follows: Denaturation at $94^{\circ} \mathrm{C}$ for $5 \mathrm{~min}$, followed by 40 amplification cycles at $94^{\circ} \mathrm{C}$ for $30 \mathrm{sec}, 56^{\circ} \mathrm{C}$ for $60 \mathrm{sec}$, and $72^{\circ} \mathrm{C}$ for $40 \mathrm{sec}$, with the final cycle extended to $10 \mathrm{~min}$ at $72^{\circ} \mathrm{C}$, and termination at $4^{\circ} \mathrm{C}$. The detection of $\beta$-actin transcripts provided an internal control in RT-PCR, standardizing the quantity of input cDNA. The quantitative RT-PCR amplification products and the expression ratios of mRNA were determined using the $2^{-\Delta \Delta \mathrm{Cq}}$ method (20) for relative quantification. The primers used in the study are listed in Table I.

Western blotting. Total proteins were extracted from PBMCs using RIPA Lysis and Extraction Buffer (Thermo Fisher Scientific, Inc.). The protein concentration was measured by BCA protein assay kit (Thermo Fisher Scientific, Inc.). Equal amounts of proteins ( $20 \mu \mathrm{g}$ per lane) were loaded onto the gel and separated by SDS-polyacrylamide gels with $10 \%$ (w/v) acrylamide, followed by electrophoresis and blotting onto PVDF membranes (EMD Millipore). The membranes were blocked in 5\% nonfat milk for $30 \mathrm{~min}$ at room temperature and co-incubated overnight with anti-NLRP3 (1:1,000; cat. no. 13158S; Cell Signaling Technology, Inc.), anti-ASC (1:1,000; cat. no. sc-514414; Santa Cruz Biotechnology, Inc.), anti-caspase-1 (1:1,000; cat. no. 3866S; Cell Signaling Technology, Inc.) and anti- $\beta$-actin (cat. no. 8457S; Cell Signaling Technology, Inc.; $1: 1,000$ ) on a shaker at $4^{\circ} \mathrm{C}$. After rinsing, the membranes were co-incubated for $2 \mathrm{~h}$ with an alkaline phosphatase-labeled goat anti rabbit secondary antibody (1:2,000; cat. no. 7054S; Cell Signaling Technology, Inc.) on a shaker at room temperature. Following additional rinsing, the membranes were treated with $5 \mathrm{ml} \mathrm{BCIP/NBT}$ solution (Invitrogen; Thermo Fisher Scientific, Inc.) according to the manufacturer's instructions. The Quantity One 1-D analysis software version 4.6.3 (Bio-Rad Laboratories, Inc.) was used 
Table I. Primers used in the present study.

\begin{tabular}{lll}
\hline Gene & \multicolumn{1}{c}{ Sequence } & Product size \\
\hline NLRP3 & & 103 bp \\
Upstream & F-5'-AAGCACCTGTTGTGCAATCTGAAG-3' & \\
Downstream & R-5'-GGGAATGGCTGGTGCTCAATAC-3' \\
ASC & & 151 bp \\
Upstream & F-5'-TGACGGATGAGCAGTACCAG-3' \\
Downstream & R-5'-TCCTCCACCAGGTAGGACTG-3' \\
Caspase-1 & \\
Upstream & F-5'-GCCTGTTCCTGTGATGTGGA-3' \\
Downstream & R-5'-TTCACTTCCTGCCCACAGAC-3' \\
$\begin{array}{l}\text { B-actin } \\
\text { Upstream }\end{array}$ & \\
Downstream & F-5'-CCTTCCTGGGCATGGAGTCCTG-3' & 175 bp \\
\end{tabular}

NLRP3, nucleotide-binding oligomerization domain, leucine rich repeat, and pyrin domain-containing protein 3; ASC, apoptosis-associated speck-like protein containing a CARD; F, forward; $R$, reverse.

to analyze the protein ladders. Protein expression levels were normalized to those of internal controls ( $\beta$-actin).

Enzyme-linked immunosorbent assay (ELISA). ELISA was used to determine the levels of IL-1 $\beta$ and IL-18 according to the manufacturer's instructions (Cell Signaling Technology, Inc.). Triplicated wells were set as samples and standard references. The final absorbance values of proteins were read by a microplate reader at $450 \mathrm{~nm}$, while the means were used to calculate IL-1 $\beta$ and IL-18 contents of corresponding samples based on the established standard curves.

Statistical analysis. The numerical data were presented as the means \pm standard deviation (SD). Statistically significant differences among the groups were evaluated by Student's t-test or one-way analysis of variance (ANOVA) followed by the Bonferroni test, using SPSS 21.0 software (IBM Corp.). Correlation analysis was performed using Pearson's correlation. P-values $<0.05$ were considered to be statistically significant.

\section{Results}

Baseline characteristics. Sixty participants were enrolled in this study and were divided into three groups [SAP $(n=20)$, AMI $(n=20)$ and non-CHD controls $(n=20)]$. No significant differences in age, sex, BMI, smoking status, mononuclear count, lipoprotein A (LPA) level, urea level, creatinine level and uric acid level were observed among the three groups ( $\mathrm{P}>0.05)$. However, total cholesterol (TC) level, triglycerides (TG) level, high-density lipoprotein cholesterol (HDL-C) level, LDL-C level, apolipoprotein B (Apo B) level and alanine transaminase (ALT) level were significantly increased in the SAP group compared to those in the control group $(\mathrm{P}<0.05)$, while white blood cell (WBC) count, HDL-C, Apo A level, ALT level, aspartate aminotransferase (AST) level and hs-CRP level were significantly increased in the AMI group compared to those in the control group $(\mathrm{P}<0.05)$. When compared to the
SAP group, the WBC count and levels of LDL-C, ALT, AST and hs-CRP in the AMI group were revealed to be significant $(\mathrm{P}<0.05)$. The baseline characteristics of the study population are presented in Table II.

Expression of NLRP3 inflammasome in PBMCs. PBMCs isolated from SAP, AMI and control participants were purified with CD14-antibody-conjugated beads, and then were identified using fluorescence-activated cell sorting (FACS) as revealed in Fig. 1A. RT-PCR results revealed that the mRNA expression of NLRP3, ASC and caspase-1 were significantly increased in SAP and AMI groups compared to the control group $(\mathrm{P}<0.05)$. The significant upregulation of the mRNA levels of the three indicators in the AMI group was concurrently revealed when compared with the SAP group $(\mathrm{P}<0.05$, Fig. 1B-D). Similar results were observed in the protein expression pattern of NLRP3 inflammasome, revealing a significant increase in the SAP and AMI group compared to the control group, and the AMI group revealed an increase compared with the SAP group $(\mathrm{P}<0.05$, Fig. $2 \mathrm{~A}$ and $\mathrm{B})$.

Measurement of $I L-1 \beta$ and $I L-18$ in plasma. As revealed in Fig. $2 \mathrm{C}$, there was a significant increase in the secretion of IL-1 $\beta$ and IL-18 in the SAP and AMI groups compared to the controls $(\mathrm{P}<0.05)$. In addition, a significant upregulation in the concentrations of the two cytokines was observed in the AMI group, compared with the SAP group $(\mathrm{P}<0.05)$.

Correlations of IL-1 $\beta$ and IL-18 levels with inflammatory markers. The correlations of cytokines IL-1 $\beta$ and IL-18 with plasma levels of the indicated inflammatory markers in a total of 60 subjects were analyzed. The results revealed that both WBC count and hs-CRP level were positively correlated with IL-1 $\beta$ and IL-18 concentrations, WBC count $(r=0.484 ; \mathrm{P}<0.05)$ and hs-CRP level $(r=0.442 ; \mathrm{P}<0.05)$ with IL-1 $\beta$, WBC count $(r=0.365 ; \mathrm{P}<0.05)$ and hs-CRP level $(r=0.293$; $\mathrm{P}=0.023)$ with IL-18. However, there were no significant correlations between 
A
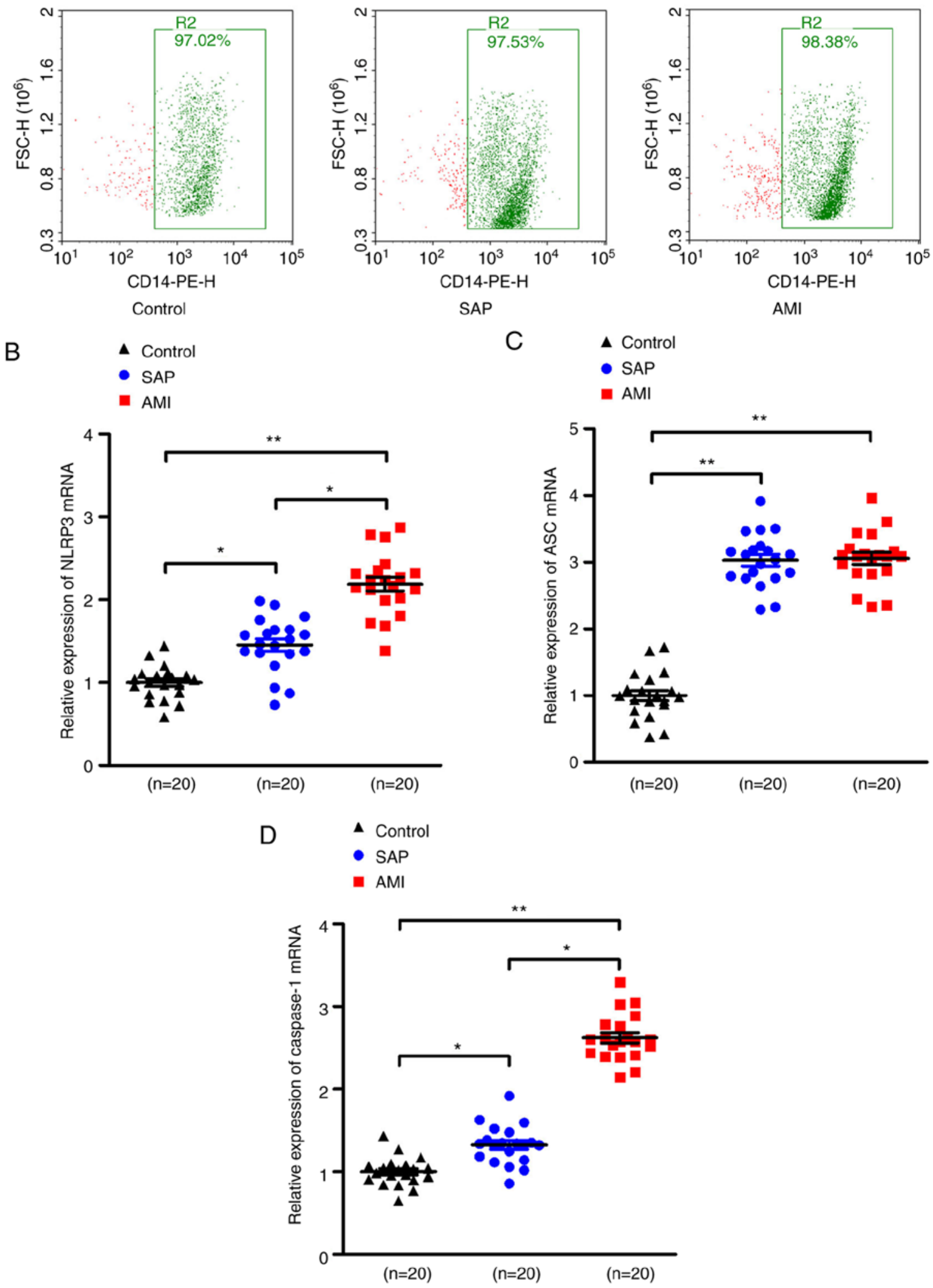

Figure 1. Upregulation of NLRP3 inflammasome mRNA levels in PBMCs in patients with SAP and AMI, compared with non-CHD controls. (A) PBMCs were isolated from peripheral blood and the positive rate of CD14 was calculated to be $\geq 95 \%$ of PBMCs by flow cytometry. RT-PCR assays were performed to quantify the mRNA levels of (B) NLRP3, (C) ASC and (D) caspase-1 in PBMCs of each group. " $\mathrm{P}<0.05$; ${ }^{* *} \mathrm{P}<0.01$. NLRP3, nucleotide-binding oligomerization domain, leucine rich repeat, and pyrin domain-containing protein 3; PBMCs, peripheral blood monocytes; SAP, stable angina pectoris; AMI, acute myocardial infarction; CHD, coronary heart disease; ASC, apoptosis-associated speck-like protein containing a CARD.

mononuclear count, Apo A level, Apo B level, or LPA level with IL-1 $\beta$ and IL-18 concentrations (Table III).
Effect of rosuvastatin on NLRP3 inflammasome and downstream cytokines in PMBCs in vitro. PBMCs isolated from 
A

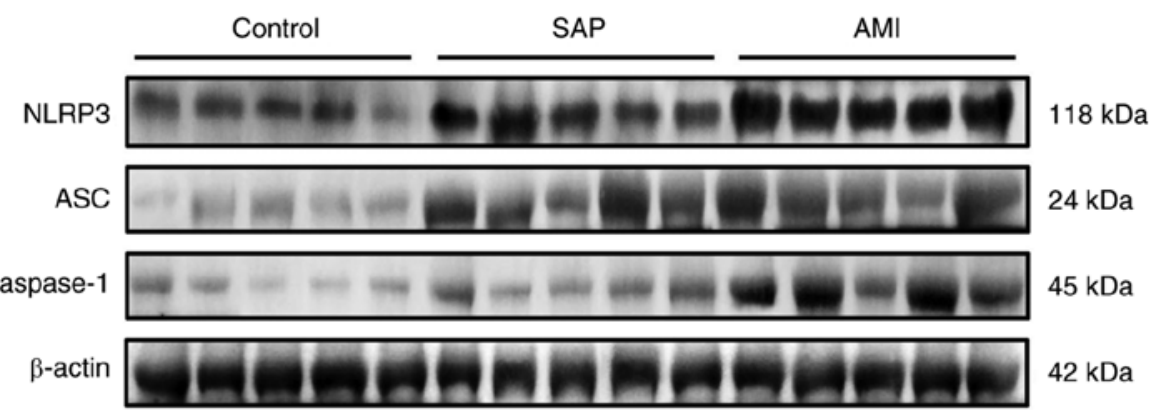

B
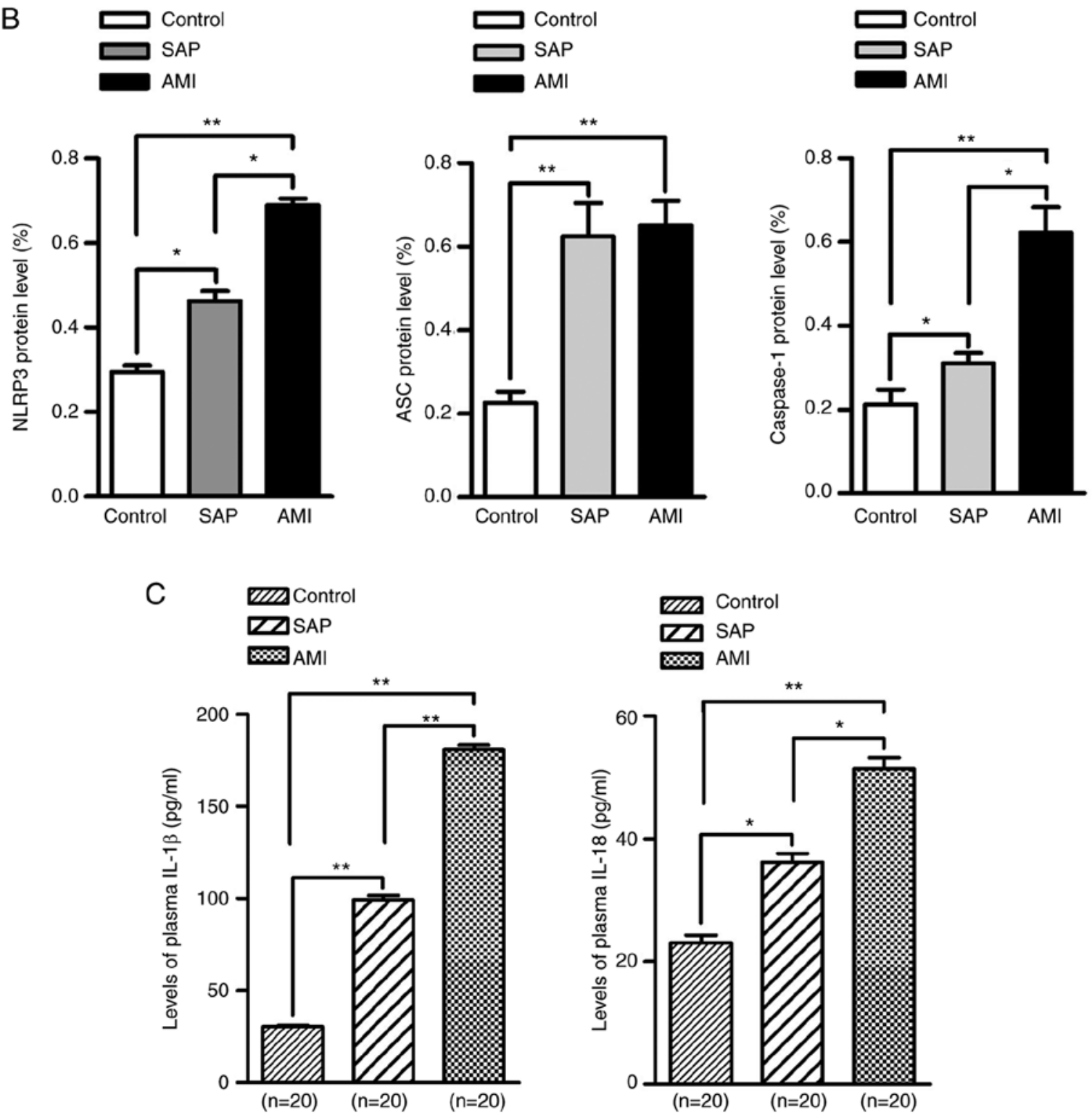

Figure 2. Increase in protein expression levels of NLRP3 inflammasome and secretion of its downstream interleukins in PBMCs of patients with SAP and AMI, compared with non-CHD controls. (A) Western blotting was used to detect the protein expression of NLRP3, ASC and caspase-1 in PBMCs of each group. (B) Quantitative results are illustrated for the three indicators using western blot analysis. (C) Plasma levels of IL-1 $\beta$ and IL-18 from each group were measured by ELISA. ${ }^{*} \mathrm{P}<0.05$; ${ }^{* *} \mathrm{P}<0.01$. NLRP3, nucleotide-binding oligomerization domain, leucine rich repeat, and pyrin domain-containing protein 3 ; PBMCs, peripheral blood monocytes; SAP, stable angina pectoris; AMI, acute myocardial infarction; CHD, coronary heart disease; ASC, apoptosis-associated speck-like protein containing a CARD; ELISA, enzyme-linked immunosorbent assay.

each group were cultured and proliferated as described in Materials and methods. Western blotting and real-time RT-PCR were performed to detect the expression of NLRP3,
ASC, and caspase-1 in PBMCs. ELISA was performed to assess IL-1 $\beta$ and IL-18 levels in the supernatants. The results revealed that the mRNA and protein expression of NLRP3, 
Table II. Baseline laboratory indices of the study group.

\begin{tabular}{|c|c|c|c|}
\hline & Control $(n=20)$ & $\operatorname{SAP}(n=20)$ & AMI (n=20) \\
\hline Age & $59.10 \pm 10.33$ & $64.05 \pm 11.52$ & $64.05 \pm 8.72$ \\
\hline BMI $\left(\mathrm{kg} / \mathrm{m}^{2}\right)$ & $24.99 \pm 3.42$ & $25.57 \pm 3.70$ & $25.7310 \pm 3.13$ \\
\hline Male & 6 & 11 & 12 \\
\hline Female & 14 & 9 & 8 \\
\hline Smoking history & $4 / 20$ & $8 / 20$ & $6 / 20$ \\
\hline CHD family history & - & $5 / 20$ & $6 / 20$ \\
\hline Hypertension & $7 / 20$ & $12 / 20$ & $10 / 20$ \\
\hline WBC (x109/1) & $5.88 \pm 1.33$ & $6.62 \pm 1.85$ & $9.59 \pm 4.44^{\mathrm{a}, \mathrm{b}}$ \\
\hline Mononuclear (x109/1) & $0.71 \pm 1.41$ & $0.44 \pm 0.13$ & $0.65 \pm 0.33$ \\
\hline $\mathrm{TC}(\mathrm{mmol} / \mathrm{l})$ & $4.54 \pm 0.96$ & $3.73 \pm 1.09^{\mathrm{a}}$ & $4.12 \pm 1.09$ \\
\hline TG (mmol/l) & $1.22 \pm 0.46$ & $1.83 \pm 1.01^{\mathrm{a}}$ & $1.51 \pm 1.11$ \\
\hline HDL (mmol/l) & $1.21 \pm 0.29$ & $0.97 \pm 0.25^{\mathrm{a}}$ & $0.97 \pm 0.25^{\mathrm{a}, \mathrm{b}}$ \\
\hline LDL (mmol/l) & $2.50 \pm 0.74$ & $1.83 \pm 0.78^{\mathrm{a}}$ & $2.50 \pm 0.92^{\mathrm{b}}$ \\
\hline Apo A $(g / l)$ & $1.33 \pm 0.35$ & $1.18 \pm 0.22$ & $1.07 \pm 0.22^{\mathrm{a}}$ \\
\hline Apo B (g/l) & $0.82 \pm 0.24$ & $0.66 \pm 0.27^{\mathrm{a}}$ & $0.80 \pm 0.25$ \\
\hline LPA (mg/l) & $205.95 \pm 228.86$ & $227.70 \pm 183.95$ & $228.10 \pm 232.37$ \\
\hline $\operatorname{ALT}(\mathrm{U} / \mathrm{l})$ & $17.35 \pm 8.80$ & $27.85 \pm 16.99^{\mathrm{a}}$ & $42.25 \pm 16.92^{\mathrm{a}, \mathrm{b}}$ \\
\hline $\operatorname{AST}(\mathrm{U} / \mathrm{l})$ & $21.50 \pm 4.48$ & $27.20 \pm 11.44$ & $146.40 \pm 132.90^{\mathrm{a}, \mathrm{b}}$ \\
\hline Urea (mmol/l) & $5.66 \pm 1.18$ & $5.62 \pm 1.34$ & $5.45 \pm 1.70$ \\
\hline Creatinine $(\mu \mathrm{mol} / \mathrm{l})$ & $63.20 \pm 11.31$ & $72.25 \pm 13.49$ & $69.85 \pm 24.20$ \\
\hline hs-CRP (mg/l) & $1.24 \pm 1.37$ & $3.34 \pm 5.74$ & $24.27 \pm 31.08^{\mathrm{a}, \mathrm{b}}$ \\
\hline Uric acid (umo1/l) & $296.10 \pm 88.46$ & $299.85 \pm 70.12$ & $297.00 \pm 86.183$ \\
\hline
\end{tabular}

${ }^{a} \mathrm{P}<0.05$ compared to the control group; ${ }^{\mathrm{b}} \mathrm{P}<0.05$ comparison between the $\mathrm{SAP}$ and AMI groups. SAP, stable angina pectoris; AMI, acute myocardial infarction; CHD, coronary heart disease; WBC, white blood cells; TC, total cholesterol; TG, triglyceride; HDL, high-density lipoprotein; LDL, low-density lipoprotein; Apo A, apolipoprotein A; Apo B, apolipoprotein B; LPA, lipoprotein A; ALT, alanine transaminase; AST, aspartate aminotransferase.

ASC and caspase-1 were downregulated in the 12 and 24-h groups treated with rosuvastatin compared to the untreated groups of PBMCs from either SAP or AMI patients in vitro $(\mathrm{P}<0.05)$, and more significant decrease was observed in the $24-\mathrm{h}$ group compared to the 12 -h group $(\mathrm{P}<0.05)$ (Figs. 3 and 4A and B). Similar results were also observed for the expression of IL-1 $\beta$ and IL-18 in the supernatants of untreated groups, and the rosuvastatin group treated for 12 and $24 \mathrm{~h}$ (Fig. 4C).

\section{Discussion}

Cardiovascular disease is still considered to be the leading cause of deaths and illnesses worldwide (1). Atherosclerosis is widely perceived as the pathological basis of CHD including SAP and AMI, wherein the cholesterol deposition incites a progressive macrophage-dominated inflammatory response. Until recently, the molecular basis of these inflammatory responses in atherosclerotic lesions was not clearly understood. In the present study, PBMCs were isolated and the expression levels of NLRP3 inflammasome and its downstream cytokines from SAP and AMI patients were identified, and the effect of rosuvastatin during this process was investigated. The results revealed that NLRP3, ASC and caspase-1 were highly expressed in PBMCs along with
Table III. Correlation of inflammatory markers with IL-1 $\beta$ and IL-18.

\begin{tabular}{lccccc}
\hline & \multicolumn{2}{c}{ IL-1 $\beta$} & & \multicolumn{2}{c}{ IL-18 } \\
\cline { 2 - 3 } \cline { 5 - 6 } Variables & $r$ & P-value & $r$ & P-value \\
WBC & 0.484 & $<0.001^{\mathrm{a}}$ & & 0.365 & $0.005^{\mathrm{a}}$ \\
Mononuclear & 0.025 & 0.849 & & 0.020 & 0.878 \\
hs-CRP & 0.442 & $<0.001^{\mathrm{a}}$ & & 0.293 & $0.023^{\mathrm{a}}$ \\
Apo A & -0.350 & $0.006^{\mathrm{a}}$ & -0.021 & 0.063 \\
Apo B & -0.039 & 0.765 & -0.053 & 0.686 \\
LPA & 0.048 & 0.717 & 0.059 & 0.656 \\
\hline
\end{tabular}

${ }^{\mathrm{a}} \mathrm{P}<0.05$. WBC, white blood cells; Apo A, apolipoprotein A; Apo B apolipoprotein $\mathrm{B}$; hs-CRP, high-sensitivity C-reactive protein; LPA, lipoprotein A.

its downstream cytokines IL-1 $\beta$ and IL-18 in SAP and AMI patients when compared to non-CHD controls. Rosuvastatin at a concentration of $20 \mu \mathrm{M}$ for 12 and $24 \mathrm{~h}$ led to a significant decrease in the expression of NLRP3 inflammasome 

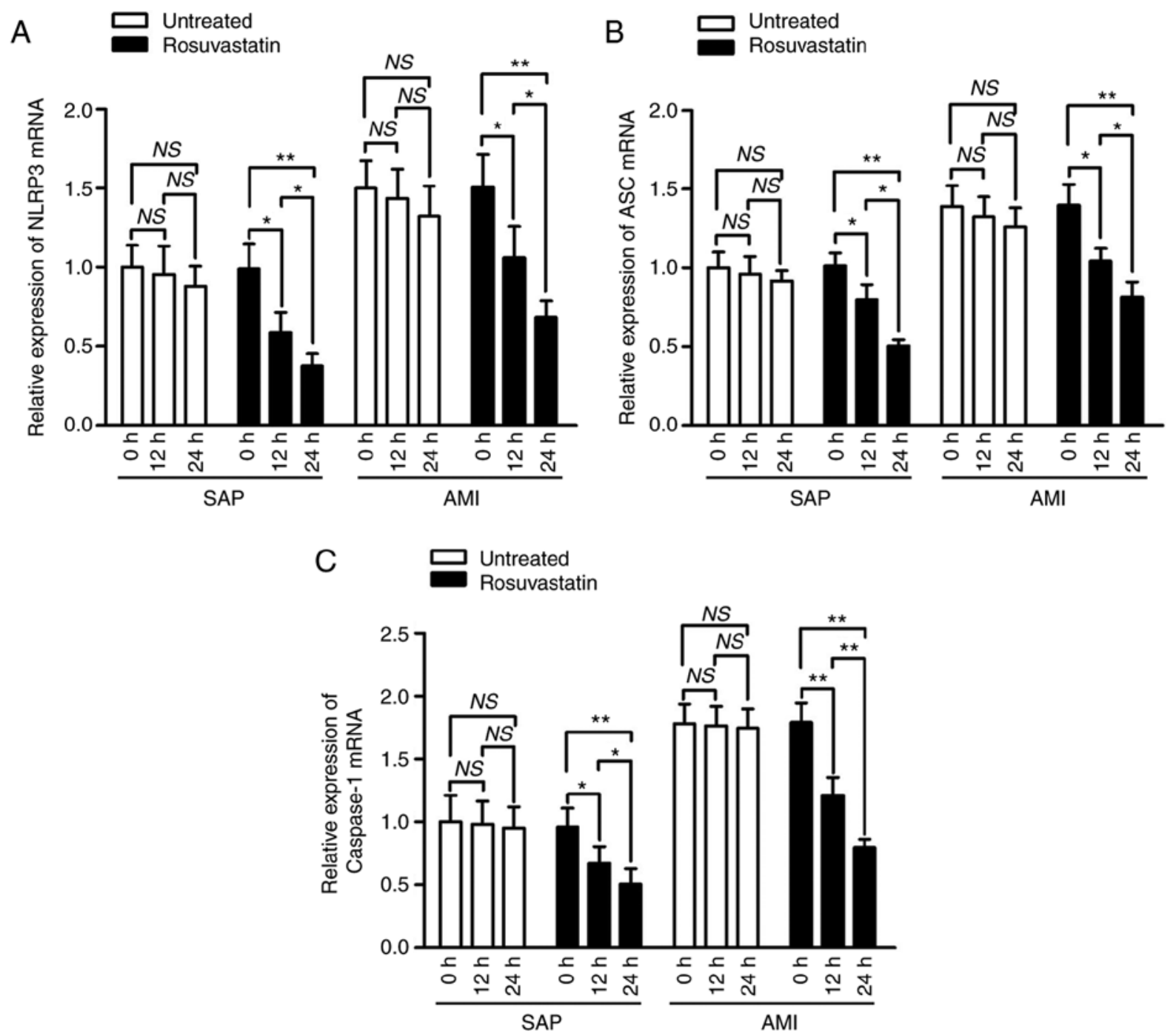

Figure 3. Effect of rosuvastatin on NLRP3 inflammasome mRNA levels in PBMCs in vitro. PBMCs were isolated from the peripheral blood of patients with non-CHD, SAP and AMI, respectively, followed by proliferation and treatment with rosuvastatin at a concentration of $20 \mu \mathrm{M}$ for the indicated time-points. RT-PCR assay was performed to investigate the influence of rosuvastatin on the mRNA levels of (A) NLRP3, (B) ASC and (C) caspase-1 in PBMCs of each group. ${ }^{*} \mathrm{P}<0.05 ;{ }^{* *} \mathrm{P}<0.01$. NLRP3, nucleotide-binding oligomerization domain, leucine rich repeat, and pyrin domain-containing protein 3 ; PBMCs, peripheral blood monocytes; CHD, coronary heart disease; SAP, stable angina pectoris; AMI, acute myocardial infarction; ASC, apoptosis-associated speck-like protein containing a CARD; NS, not significant.

and its downstream mediators in vitro in a time-dependent manner.

Atherosclerosis is an inflammatory condition that involves both acute and chronic components. Recent insights indicated that innate immune cells play a vital role in the pathogenesis of atherosclerosis (21). Atherosclerotic plaque development in the arterial wall is characterized by infiltration of monocytes (22). Circulating monocytes initially adhere to the activated endothelium, then infiltrate into the vessel wall via endothelial cell junction, form lesional macrophages, and then participate decisively in the development and exacerbation of atherosclerosis. The inflammatory cytokines are secreted to modify the endothelial function, proliferation of vascular smooth muscle cell (VSMCs), degradation of collagen, and thrombosis (23). The inflammasomes are innate immune system receptors/sensors that are formed in response to infectious microbes and molecules derived from host proteins (24). It is a cytoplasmic macromolecular protein complex that contains multiple proteins, which is formed in response to danger-associated molecular patterns (DAMPs) or pathogen-associated molecular patterns (PAMPs), and serves as a molecular platform for activation of cysteine protease caspase-1 (25). NLRP3 inflammasome is one of the most studied inflammasomes, and involved in the process of sterile inflammation (26).

It is well known that an array of exogenous and endogenous stimuli can activate NLRP3 inflammasome, and the underlying precise mechanism remains unclear. However, several common upstream pathways have been identified including potassium $\left(\mathrm{K}^{+}\right)$efflux (27), the generation of mitochondrial reactive oxygen species (ROS) (28), and cathepsin release as a result of phagolysosomal membrane destabilization (29). NLRP3 activation forms a complex, which has been revealed to regulate the activation of caspase- 1 and promote the production of inflammatory cytokines, such as IL-1 $\beta$ and IL-18 (30). Clinical studies have revealed that the increase in IL-1 $\beta$ and IL-18 levels is related to clinical severity in 
A
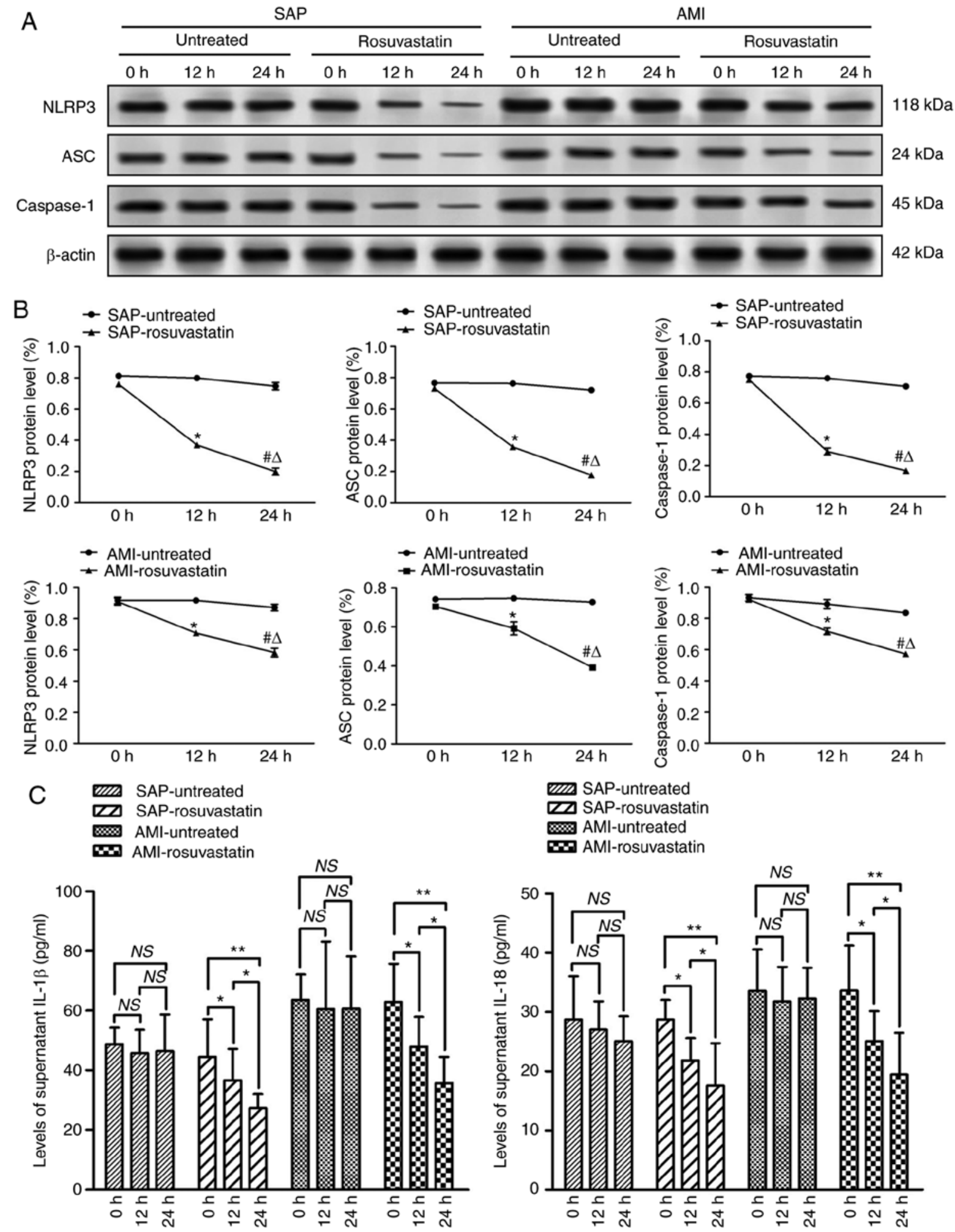

Figure 4. Effect of rosuvastatin on protein expression levels of NLRP3 inflammasome in PBMCs and secretion of its downstream cytokines in supernatants in vitro. PBMCs were isolated from the peripheral blood of patients with non-CHD, SAP and AMI, respectively, followed by proliferation and treatment with rosuvastatin at a concentration of $20 \mu \mathrm{M}$ for the indicated time-points. (A) Western blotting was conducted to analyze the influence of rosuvastatin on the protein levels of NLRP3, ASC and caspase-1 in PBMCs of each group. (B) Quantitative results for modulating the protein expression of the aforementioned three indicators by rosuvastatin are illustrated. (C) Modulation of IL-1 $\beta$ and IL-18 secretion in the supernatants by rosuvastatin of each group was measured by ELISA. " $\mathrm{P}<0.05,{ }^{* *} \mathrm{P}<0.01$ and ${ }^{\#} \mathrm{P}<0.05$ (compared to the 0 -h group); ${ }^{\Delta} \mathrm{P}<0.05$ (compared to the 12 -h group). NLRP3, nucleotide-binding oligomerization domain, leucine rich repeat, and pyrin domain-containing protein 3; PBMCs, peripheral blood monocytes; CHD, coronary heart disease; SAP, stable angina pectoris; AMI, acute myocardial infarction; ASC, apoptosis-associated speck-like protein containing a CARD; NS, not significant.

patients with CHD $(31,32)$. In recent years, NLRP3 inflammasome has been considered as a novel player in myocardial infarction (33). Animal experiments were performed to identify whether inflammasome activation of cardiac fibroblasts 
remains essential for myocardial ischemia/reperfusion injury (34). Consistent with this, a study revealed that inhibition of NLRP3 inflammasome limited the inflammatory injury following myocardial ischemia-reperfusion in a mouse model (35). Therefore, NLRP3 inflammasome and associated IL- $1 \beta$ release are considered as potential biomarkers of conventional cardiovascular risk (36). CRP, as an important inflammatory mediator, is able to directly participate in the pathogenesis of atherosclerosis by activating endothelial cells and promoting the inflammatory component of atherosclerosis $(37,38)$. Moreover, the circulating level of hs-CRP was also revealed to be strongly associated with the clinical setting of unstable angina pectoris $(39,40)$. In the present study, peripheral blood was collected from patients within $12 \mathrm{~h}$ after an attack of AMI, and emergency PCI was applied to testify and treat the target lesion. It was revealed that WBC count, levels of hs-CRP, NLRP 3 inflammasome and its downstream cytokines IL-1 $\beta$ and IL-18 were all significantly increased in patients with AMI when compared to non-CHD controls. Expression of key factors of the NLRP3 inflammasome signaling pathway were also observed to be enhanced in SAP patients.

Furthermore, it was determined that IL-1 $\beta$ and IL-18 content revealed a correlation with WBC count and the level of hs-CRP. These findings revealed an inflammatory response in coronary artery occlusion, especially in the process of AMI characterized by a greater and a more marked activation of inflammation. The results that the increase in either hs-CRP level or WBC count in SAP patients was not significant enough when compared to non-CHD controls also support the notion. In light of this evidence, it is proposed that the stimulated PBMCs orchestrate the activation of NLRP3 inflammasome. This was due to the upregulation of the expression of NLRP3, ASC, caspase-1, and their downstream mediators, leading to atherosclerotic progression (Fig. 5).

The elevated blood cholesterol levels, or more precisely the LDL-C levels, are generally recognized as a major risk factor and are causally linked to the pathogenesis of atherosclerosis (41). Rosuvastatin has been extensively used for the treatment of hyperlipidemia and CHD, and is mainly referred due to its benefit to stabilization and regression of atherosclerotic plaques, which thus avoids or improves cardiovascular events. Recently, rosuvastatin was also reported to have the ability to decrease the number of inflammatory cells in atherosclerotic plaques and possess other anti-inflammatory properties (42). The potential relationship between rosuvastatin and NLRP3 inflammasome in coronary artery disease (CAD) has been documented by previous studies $(8,43)$, however, SAP and AMI represent different stages of development of coronary atherosclerosis with different pathophysiological processes, and their underlying molecular mechanisms are far from complete.

In the present study, $\mathrm{CD} 14^{+}$monocytes were cultured and treated with rosuvastatin at different time-points in vitro, and it was revealed that these indicators were significantly downregulated in a time-dependent manner in patients with SAP and AMI. However, in patients without CHD, the effect of rosuvastatin exhibited no significant results. These findings indicated an activation of the NLRP3 inflammasome signaling pathway during the inflammatory process of atherosclerosis.

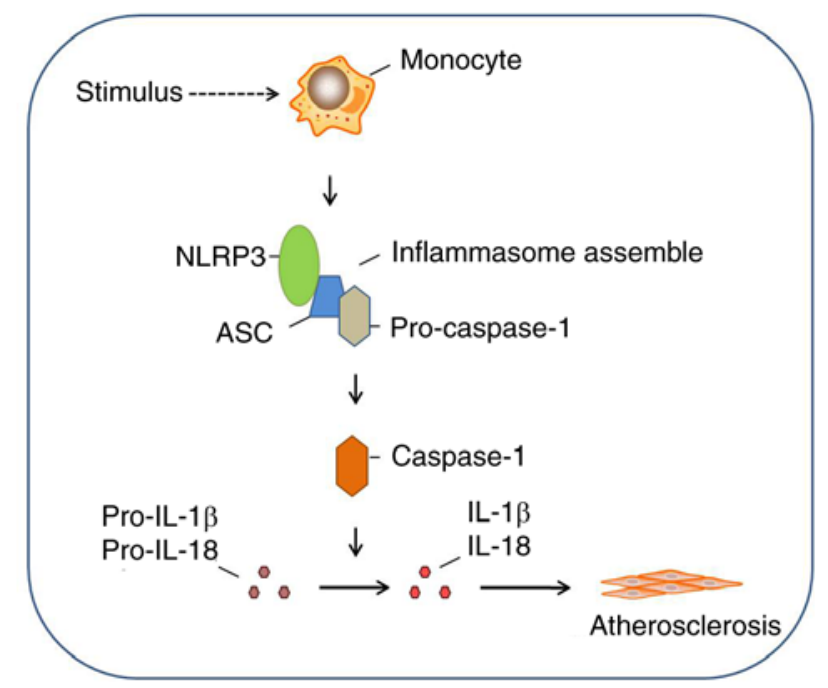

Figure 5. Proposed model for NLRP3 inflammasome signaling pathway involved in inflammatory pathogenesis of coronary atherosclerosis. Stimulated PBMCs orchestrate activation of NLRP3 inflammasome due to the upregulation of the expression of NLRP3, ASC, caspase-1, and their downstream mediators, leading to atherosclerotic progression. NLRP3, nucleotide-binding oligomerization domain, leucine rich repeat, and pyrin domain-containing protein 3; PBMCs, peripheral blood monocytes; ASC, apoptosis-associated speck-like protein containing a CARD.

Rosuvastatin could partially reverse the activation of this pathway in different stages of atherogenesis, particularly in the development of stable plaques including plaque rupture. Based on the aforementioned findings, the present study confirmed the anti-inflammatory effect of rosuvastatin, which was independent of lipid-level lowering.

In summary, it was revealed that PBMCs from patients with either SAP or AMI have a higher expression of NLRP3 inflammasome and its downstream cytokines, and activation of these indicators revealed a correlation particularly in AMI patients. Rosuvastatin exhibited desirable inhibitory effects on the activation of the NLRP3 inflammasome signaling pathway in a time-dependent manner. These findings indicated NLRP3 inflammasome as a potential target in the inflammatory process that leads to atherosclerosis, and provided a new pharmacological role of rosuvastatin as an anti-inflammatory drug for CHD including SAP and AMI.

\section{Acknowledgements}

Not applicable.

\section{Funding}

The present study was supported by a grant from the Natural Science Research Key Project of Education Office of Anhui Province (grant no. KJ2018A0242), and an internal grant from Bengbu Medical College (grant no. BYKY1672).

\section{Availability of data and materials}

The datasets used and/or analyzed during the present study are available from the corresponding author upon reasonable request. 


\section{Authors' contributions}

JZ, SW, SH and HW designed the experiments. JZ, SW and HL performed the experiments. JZ, ML and XG analyzed the data. JZ, SW and SH wrote the manuscript. JZ and HW revised the manuscript. All authors read, reviewed and approved the final manuscript.

\section{Ethics approval and consent to participate}

The utilization of samples was approved by the Ethics Committee of The First Affiliated Hospital of Bengbu Medical College (Bengbu, China) and written informed consent was obtained from all subjects.

\section{Patient consent for publication}

Not applicable.

\section{Competing interests}

The authors declare that they have no competing interests.

\section{References}

1. Benjamin EJ, Blaha MJ, Chiuve SE, Cushman M, Das SR Deo R, de Ferranti SD, Floyd J, Fornage M, Gillespie C, et al: Heart disease and stroke statistics-2017 update: A report from the American heart association. Circulation 135: e146-e603, 2017.

2. Welsh P, Grassia G, Botha S, Sattar N and Maffia P: Targeting inflammation to reduce cardiovascular disease risk: A realistic clinical prospect? Br J Pharmacol 174: 3898-3913, 2017.

3. Ridker PM, Everett BM, Thuren T, MacFadyen JG, Chang WH, Ballantyne C, Fonseca F, Nicolau J, Koenig W, Anker SD, et al Antiinflammatory therapy with canakinumab for atherosclerotic disease. N Engl J Med 377: 1119-1131, 2017.

4. Ridker PM, Hennekens CH, Buring JE and Rifai N: C-reactive protein and other markers of inflammation in the prediction of cardiovascular disease in women. N Engl J Med 342: 836-843, 2000.

5. Duewell P, Kono H, Rayner KJ, Sirois CM, Vladimer G, Bauernfeind FG, Abela GS, Franchi L, Nuñez G, Schnurr M, et al: NLRP3 inflammasomes are required for atherogenesis and activated by cholesterol crystals. Nature 464: 1357-1361, 2010.

6. Kingsbury SR, Conaghan PG and McDermott MF: The role of the NLRP3 inflammasome in gout. J Inflamm Res 4: 39-49, 2011.

7. Toldo S, Mezzaroma E, Mauro AG, Salloum F and Van Tassell BW and Abbate A: The inflammasome in myocardial injury and cardiac remodeling. Antioxid Redox Signal 22: 1146-1161, 2015.

8. Satoh M, Tabuchi T, Itoh T and Nakamura M: NLRP3 inflammasome activation in coronary artery disease: Results from prospective and randomized study of treatment with atorvastatin or rosuvastatin. Clin Sci (Lond) 126: 233-241, 2014.

9. Schroder K, Zhou R and Tschopp J: The NLRP3 inflammasome: A sensor for metabolic danger? Science 327: 296-300, 2010.

10. Rajamäki K, Lappalainen J, Oörni K, Välimäki E, Matikainen S, Kovanen PT and Eklund KK: Cholesterol crystals activate the NLRP3 inflammasome in human macrophages: A novel link between cholesterol metabolism and inflammation. PLoS One 5: e11765, 2010

11. No authors listed: Randomised trial of cholesterol lowering in 4444 patients with coronary heart disease: The scandinavian simvastatin survival study (4S). Lancet 344: 1383-1389, 1994.

12. Ridker PM, Danielson E, Fonseca FA, Genest J, Gotto AM Jr, Kastelein JJ, Koenig W, Libby P, Lorenzatti AJ, MacFadyen JG, et al: Rosuvastatin to prevent vascular events in men and women with elevated C-reactive protein. N Engl J Med 359: 2195-2207, 2008.
13. Schwartz GG, Olsson AG, Ezekowitz MD, Ganz P, Oliver MF, Waters D, Zeiher A, Chaitman BR, Leslie S and Stern T; Myocardial Ischemia Reduction with Aggressive Cholesterol Lowering (MIRACL) Study Investigators: Effects of atorvastatin on early recurrent ischemic events in acute coronary syndromes: The MIRACL study: A randomized controlled trial. JAMA 285: 1711-1718, 2001.

14. Zacà V, Rastogi S, Imai M, Wang M, Sharov VG, Jiang A, Goldstein S and Sabbah HN: Chronic monotherapy with rosuvastatin prevents progressive left ventricular dysfunction and remodeling in dogs with heart failure. J Am Coll Cardiol 50: 551-557, 2007.

15. Ren Y, Zhu H, Fan Z, Gao Y and Tian N: Comparison of the effect of rosuvastatin versus rosuvastatin/ezetimibe on markers of inflammation in patients with acute myocardial infarction. Exp Ther Med 14:4942-4950, 2017.

16. Karlson BW, Nicholls SJ, Lundman P, Barter PJ and Palmer MK: Modeling statin-induced reductions of cardiovascular events in primary prevention: A VOYAGER meta-Analysis. Cardiology 140: 30-34, 2018.

17. FihnSD, GardinJM,Abrams J,BerraK,Blankenship JC,Dallas AP, Douglas PS, Foody JM, Gerber TC, Hinderliter AL, et al: 2012 ACCF/AHA/ACP/AATS/PCNA/SCAI/STS guideline for the diagnosis and management of patients with stable ischemic heart disease: Executive summary: A report of the American college of cardiology foundation/American heart association task force on practice guidelines, and the American College of physicians, American association for thoracic surgery, preventive cardiovascular nurses association, society for cardiovascular angiography and interventions, and society of thoracic surgeons. Circulation 126: 3097-3137, 2012.

18. Thygesen K, Alpert JS, Jaffe AS, Simoons ML, Chaitman BR, White HD; Joint ESC/ACCF/AHA/WHF Task Force for the Universal Definition of Myocardial Infarction, Katus HA Lindahl B, Morrow DA, et al: Third universal definition of myocardial infarction. Circulation 126: 2020-2035, 2012.

19. Giampietro C, Lionetti MC, Costantini G, Mutti F, Zapperi S and La Porta CA: Cholesterol impairment contributes to neuroserpin aggregation. Sci Rep 7: 43669, 2017.

20. Livak KJ and Schmittgen TD: Analysis of relative gene expression data using real-time quantitative PCR and the 2(-Delta Delta C(T)) method. Methods 25: 402-408, 2001.

21. Marchant DJ, Boyd JH, Lin DC, Granville DJ, Garmaroudi FS and McManus BM: Inflammation in myocardial diseases. Circ Res 110: 126-144, 2012.

22. Weber C and Soehnlein O: ApoE controls the interface linking lipids and inflammation in atherosclerosis. J Clin Invest 121: 3825-3827, 2011.

23. Husain K, Hernandez W, Ansari RA and Ferder L: Inflammation, oxidative stress and renin angiotensin system in atherosclerosis. World J Biol Chem 6: 209-217, 2015.

24. Guo H, Callaway JB and Ting JP: Inflammasomes: Mechanism of action, role in disease, and therapeutics. Nat Med 21: 677-687, 2015.

25. Martinon F, Burns K and Tschopp J: The inflammasome: A molecular platform triggering activation of inflammatory caspases and processing of proIL-beta. Mol Cell 10: 417-426, 2002.

26. Lamkanfi $M$ and Dixit VM: Mechanisms and functions of inflammasomes. Cell 157: 1013-1022, 2014.

27. Muñoz-Planillo R, Kuffa P, Martinez-Colón G, Smith BL, Rajendiran TM and Núñez G: $\mathrm{K}^{+}$efflux is the common trigger of NLRP3 inflammasome activation by bacterial toxins and particulate matter. Immunity 38: 1142-1153, 2013.

28. Alfonso-Loeches S, Ureña-Peralta JR, Morillo-Bargues MJ, Oliver-De La Cruz J and Guerri C: Role of mitochondria ROS generation in ethanol-induced NLRP3 inflammasome activation and cell death in astroglial cells. Front Cell Neurosci 8: 216, 2014.

29. Hornung V, Bauernfeind F, Halle A, Samstad EO, Kono H, Rock KL, Fitzgerald KA and Latz E: Silica crystals and aluminum salts activate the NALP3 inflammasome through phagosomal destabilization. Nat Immunol 9: 847-856, 2008.

30. Toldo S and Abbate A: The NLRP3 inflammasome in acute myocardial infarction. Nat Rev Cardiol 15: 203-214, 2018.

31. Galea J, Armstrong J, Gadsdon P, Holden H, Francis SE and Holt CM: Interleukin-1 beta in coronary arteries of patients with ischemic heart disease. Arterioscler Thromb Vasc Biol 16: 1000-1006, 1996.

32. Blankenberg S, Tiret L, Bickel C, Peetz D, Cambien F, Meyer J and Rupprecht HJ; AtheroGene Investigators: Interleukin-18 is a strong predictor of cardiovascular death in stable and unstable angina. Circulation 106: 24-30, 2002. 
33. Takahashi M: NLRP3 inflammasome as a novel player in myocardial infarction. Int Heart J 55: 101-105, 2014.

34. Kawaguchi M, Takahashi M, Hata T, Kashima Y, Usui F, Morimoto H, Izawa A, Takahashi Y, Masumoto J, Koyama J, et al: Inflammasome activation of cardiac fibroblasts is essential for myocardial ischemia/reperfusion injury. Circulation 123: 594-604, 2011.

35. Toldo S, Marchetti C, Mauro AG, Chojnacki J, Mezzaroma E, Carbone S, Zhang S, Van Tassell B, Salloum FN and Abbate A: Inhibition of the NLRP3 inflammasome limits the inflammatory injury following myocardial ischemia-reperfusion in the mouse. Int J Cardiol 209: 215-220, 2016.

36. Bullón P, Cano-García FJ, Alcocer-Gómez E, Varela-López A, Roman-MaloL,Ruiz-SalmerónRJ,Quiles JL, Navarro-PandoJM, Battino M, Ruiz-Cabello J, et al: Could NLRP3-inflammasome be a cardiovascular risk biomarker in acute myocardial infarction patients? Antioxid Redox Signal 27: 269-275, 2017.

37. Verma S, Buchanan MR and Anderson TJ: Endothelial function testing as a biomarker of vascular disease. Circulation 108 2054-2059, 2003.

38. Szmitko PE, Wang CH, Weisel RD, de Almeida JR, Anderson TJ and Verma S: New markers of inflammation and endothelial cell activation: Part I. Circulation 108: 1917-1923, 2003.

39. Yip HK, Wu CJ, Hang CL, Chang HW, Yang CH, Hsieh YK, Fang $\mathrm{CY}, \mathrm{Fu} \mathrm{M}$, Yeh $\mathrm{KH}$ and Chen MC: Levels and values of inflammatory markers in patients with angina pectoris. Int Heart J 46: 571-581, 2005.
40. Fiechter M, Ghadri JR, Jaguszewski M, Siddique A, Vogt S, Haller RB, Halioua R, Handzic A, Kaufmann PA, Corti R, et al: Impact of inflammation on adverse cardiovascular events in patients with acute coronary syndromes. J Cardiovasc Med (Hagerstown) 14: 807-814, 2013.

41. Ference BA, Ginsberg HN, Graham I, Ray KK, Packard CJ, Bruckert E, Hegele RA, Krauss RM, Raal FJ, Schunkert H, et al: Low-density lipoproteins cause atherosclerotic cardiovascular disease. 1. Evidence from genetic, epidemiologic, and clinical studies. A consensus statement from the European atherosclerosis society consensus panel. Eur Heart J 38: 2459-2472, 2017.

42. Tousoulis D, Andreou I, Tsiatas M, Miliou A, Tentolouris C, Siasos G, Papageorgiou N, Papadimitriou CA, Dimopoulos MA and Stefanadis C: Effects of rosuvastatin and allopurinol on circulating endothelial progenitor cells in patients with congestive heart failure: The impact of inflammatory process and oxidative stress. Atherosclerosis 214: 151-157, 2011.

43. Altaf A, Qu P, Zhao Y, Wang H, Lou D and Niu N: NLRP3 inflammasome in peripheral blood monocytes of acute coronary syndrome patients and its relationship with statins. Coron Artery Dis 26: 409-421, 2015.

This work is licensed under a Creative Commons

Attribution-NonCommercial-NoDerivatives 4.0 International (CC BY-NC-ND 4.0) License. 